\title{
烷基铝试剂与亲电试剂的偶联反应研究进展
}

\author{
李清寒* 罗瑞强吴川肖红柳郭少鹏 \\ 张志豪黄哲耀周林 \\ (西南民族大学化学与环境保护工程学院 成都 610041)
}

\begin{abstract}
摘要 烷基铝试剂因其反应活性高、毒性低、易于制备，广泛应用于有机反应中. 过渡金属催化或无催化剂的条件下， 有机铝试剂与亲电试剂的交叉偶联反应为多种化合物的合成提供了一种简便的方法, 并显示出比有机锂和有机镁试剂 更高的官能团耐受性，可以在硝基、酯基、羟基、氨基、腈基和内酯的存在下与羰基进行加成反应，与亲电试剂进行 偶联反应. 近年来许多有机铝试剂在交叉偶联反应中得到了较广泛的应用. 综述了近年来烷基铝试剂在交叉偶联反应 中的研究成果, 涉及到各种反应体系.
\end{abstract}

关键词＼cjkstart烷基铝试剂; 亲电试剂; 偶联反应; 碳碳键形成; 碳-杂原子键形成; 过渡金属; 无催化剂

\section{Research Progress of Cross-Coupling Reactions of Alkylaluminums with Electrophiles Reagents}

\author{
Li, Qinghan* \\ Luo, Ruiqiang \\ $\mathrm{Wu}$, Chuan \\ Xiao, Hongliu \\ Guo, Shaopeng \\ Zhang, Zhihao \\ Huang, Zheyao \\ Zhou, Lin \\ (College of Chemistry and Environmental Protection Engineering, Southwest Minzu University, Chengdu 610041)
}

\begin{abstract}
Alkyl aluminum compounds are widely applied in organic reactions because of their high reactivities, low toxicities, and ease of preparation. The cross-coupling reaction of an organoalane compounds with organic electrophiles using a transition-metal catalyst or catalyst-free provides a simple method to synthesize a large variety of compounds and shows a higher functional group tolerance than organolithium and magnesium, which allows for additions to aldehydes in the presence of nitro, ester, hydroxyl, amino, nitrile and lactone moieties. Therefore, many organoalane reagents have found the most applications in cross-coupling reactions in recent years. In this paper, the recent research results about the alkylaluminum reagents applied in cross-coupling reactions are reviewed, involving various reaction systems.

Keywords alkylaluminum reagents; electrophilic reagents; cross-coupling reaction; carbon-carbon bond formation; carbonheteroatom bond formation; transition-metal catalyst; catalyst-free
\end{abstract}

自从诺贝尔化学奖授予研究钯催化的交叉偶联反 应的先驱之后，碳-碳键形成反应成为了有机化学研究 的一个热点领域. 硼、镁、锡、锌和钛等金属有机试剂 是交叉偶联反应中最常用的亲核试剂[1-8]，然而使用有 机铝试剂进行的交叉偶联反应往往被忽视 ${ }^{[9]}$. 有机铝试 剂因其具有成本低、易得、毒性小、较强的路易斯酸性 和强亲核性的优点, 在有机合成和金属有机化学中占有 重要的地位 ${ }^{[9-12]}$. 有机铝试剂与亲电试剂在过渡金属催 化下的交叉偶联反应为多种化合物的制备提供了一类
简便的方法，并且显示出比有机锂和有机镁试剂更高的 官能团耐受性. 因此，近年来许多有机铝试剂在交叉偶 联反应中得到了广泛的应用. 我们已对烯基铝试剂、炔 基铝试剂和芳基铝试剂在交叉偶联反应中的应用研究 进展进行了综述 ${ }^{[11-13]}$, 本文将重点介绍烷基铝试剂作为 亲核试剂在过渡金属催化剂和无催化剂体系中进行交 叉偶联反应的研究进展. 其中金属钯催化烷基铝试剂参 与的交叉偶联反应内容已在我们之前的一篇综述文章 中进行了介绍 ${ }^{[14]}$, 这里主要介绍过渡金属镍、铜、铁、

\footnotetext{
* Corresponding author. E-mail: lqhchem@163.com

Received September 12, 2020; revised October 13, 2020; published online November 3, 2020

Project supported by the Fundamental Research Funds for the Central Universities, the Southwest Minzu University (No. 2018NZD06) and the Sichuan Provincial Department of Science and Technology Support Program (No. 2015NZ0033).

西南民族大学中央高校基本科研业务费(No. 2018NZD06)、四川省科技厅科技支撑计划(No. 2015NZ0033)资助项目.
} 
钴、铝等催化体系及无催化剂体系.

\section{1 金属催化体系}

\section{1 金属镍催化体系}

虽然金属钯催化剂已成功地应用于许多交叉偶联 反应中, 但近年来, 成本较低的镍催化剂在交叉偶联反 应中也表现出优异的催化性能 ${ }^{[15-17]}$.

2017 年, 王忠夏和他的合作者 ${ }^{[18]}$ 报道了三烷基铝 试剂与芳基三甲基铵的交叉偶联反应(Eq. 1). 作者使用 $5 \mathrm{~mol} \% \mathrm{Ni}(\mathrm{dppp}) \mathrm{Cl}_{2}$ 为催化剂, 在二氧六环中, 三甲基 芳基铵(2)与 $\mathrm{AlMe}_{3}$ 和具有 $\beta-\mathrm{H}$ 的三烷基铝(1)试剂于 $110{ }^{\circ} \mathrm{C}$ 反应 $24 \mathrm{~h}$, 以 $20 \% \sim 98 \%$ 的收率得到烷基芳基偶 联产物 3. 具有荎基、葱基和取代苯基三甲基的铵盐可 用于该催化体系，同时，该催化剂体系还适用于三甲基 (2-甲基萘基)铵与 $\mathrm{AlMe}_{3}$ 、三甲基(1-萗基)铵与三芳基铝 试剂的交叉偶联反应, 并取得了良好的反应效果. 但是 在相同条件下，三甲基(2-甲基䒺基)铵与含 $\beta$ - $\mathrm{H}$ 的三烷 基铝试剂如 $\mathrm{AlEt}_{3}$ 和 $\mathrm{Al}\left({ }^{n} \mathrm{C}_{8} \mathrm{H}_{17}\right)_{3}$ 反应, 产物收率相对较 低. 对于含有三氟甲基、三氟甲氧基和 1-吲哚基等官能 团的亲电试剂在该反应中兼容, 对于含有羰基官能团如 $\mathrm{COOEt}$ 和 $\mathrm{PhC}=\mathrm{O}$ 的底物则不能很好地兼容. 当使用 10 mol\% $\mathrm{Ni}(\mathrm{dppp}) \mathrm{Cl}_{2}$ 催化 $p$-[BocN(Me) $] \mathrm{C}_{6} \mathrm{H}_{4} \mathrm{NMe}_{3}{ }^{+} \mathrm{OTf}^{-}$分 别与 $\mathrm{AlMe}_{3}$ 和 $\mathrm{AlEt}_{3}$ 反应时, 分别以 $38 \%$ 和 $33 \%$ 的收率 得到脱 Boc 的偶联产物.

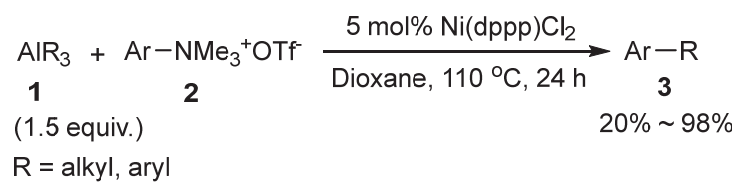

根据已报道的过渡金属催化有机金属试剂的交叉 偶联反应的研究结果 ${ }^{[19-20]}$, 作者对该反应提出了一种可 能的催化循环机制，如图 1 所示.

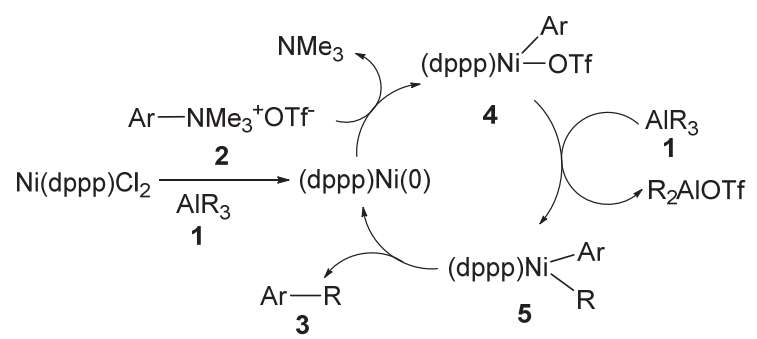

图 1 镍催化芳基三甲基三氟磺酸铵盐与有机铝试剂的交叉 偶联反应的可能机理

Figure 1 Proposed mechanism of the cross-coupling reaction of aryltrimethylammonium triflates with organoalanes use nickel as catalyst

2018 年, Kimura 和他的同事 ${ }^{[21]}$ 报道了镍催化末端 烯烃、二氧化碳及烷基铝试剂的交叉偶联反应(Eq. 2).
作者使用 $5 \mathrm{~mol} \% \mathrm{Ni}(\mathrm{cod})_{2} / 10 \mathrm{~mol} \% \mathrm{PCy}_{3}$ 为催化剂，二 氧六环为溶剂, 于 $40{ }^{\circ} \mathrm{C}$ 反应 $24 \mathrm{~h}$, 末端烯烃 6 能与 $\mathrm{AlMe}_{3}$ (7)和二氧化碳顺利地进行交叉偶联反应，并且 以中等到良好的收率得到烯丙基醇 $(\mathbf{8})$ 化合物，反应具 有良好的区域选择性和立体选择性。然而，使用 ${ }^{i} \mathrm{PrAlEt}_{2}$ 和 $\mathrm{PhCH}_{2} \mathrm{AlEt}_{2}$ 时并没有得到烯丙基醇类化合物, 而是分别以 $51 \%$ 和 $34 \%$ 的收率得到三烯丙基甲醇和三 苄基甲醇.

$$
\begin{aligned}
& \mathrm{R}_{6}^{\mathrm{R}^{2}}+\mathrm{CO}_{2}+\underset{\mathrm{REt}_{2} \mathrm{Al}}{\mathrm{Ror}^{3} \mathrm{Al}} \frac{\begin{array}{c}
5 \mathrm{~mol} \% \mathrm{Ni}(\mathrm{cod})_{2} \\
10 \mathrm{~mol} \% \mathrm{PCy}_{3}
\end{array}}{1,4 \text {-dioxane, } 40^{\circ} \mathrm{C}, 24 \mathrm{~h}} \\
& \overbrace{8}^{\mathrm{R}_{\mathrm{R}}^{2}} \overbrace{\mathrm{R}_{\mathrm{R}}^{\mathrm{OH}}}^{\mathrm{R}}+\mathrm{X}_{\mathrm{R}}^{\mathrm{OH}} \\
& \mathrm{Me}_{3} \mathrm{Al} \quad 24 \% \sim 86 \% \\
& { }^{i} \mathrm{PrAlEt}_{2} \\
& \mathrm{PhCH}_{2} \mathrm{AlEt}_{2} \quad 34 \% \\
& \mathrm{R}^{1}=\text { alkyl, aryl, 3-indoly, naphthyl; } \mathrm{R}^{2}=\mathrm{H}, \mathrm{Me}, \mathrm{Ph}
\end{aligned}
$$

2019 年, 王朝諺和他的同事 ${ }^{[22]}$ 报道了镍催化(杂)芳 基醚和烷基铝试剂的交叉偶联反应(Eq. 3). 他们发现， 在 $130{ }^{\circ} \mathrm{C}$ 以甲苯为溶剂，用 $10 \mathrm{~mol} \% \mathrm{Ni}(\operatorname{cod})_{2} / 10 \mathrm{~mol} \%$ dcype 能有效催化芳基醚或甲氧基吡啶(10)与 $\mathrm{Me}_{3} \mathrm{Al}$ (11)的交叉偶联反应. 在标准加热条件下，所有交叉偶 联反应均可在 $18 \mathrm{~h}$ 内完成, 其偶联产物 12 的收率达 $99 \%$. 此外, $\mathrm{Et}_{3} \mathrm{Al} 、\left(\mathrm{C}_{8} \mathrm{H}_{17}\right)_{3} \mathrm{Al}$ 和 $\left(\mathrm{CH}_{2} \mathrm{Ph}\right)_{3} \mathrm{Al}$ 也适用于该 反应体系，并以 $85 \% \sim 91 \%$ 的良好收率得到相应的偶联 产物 12

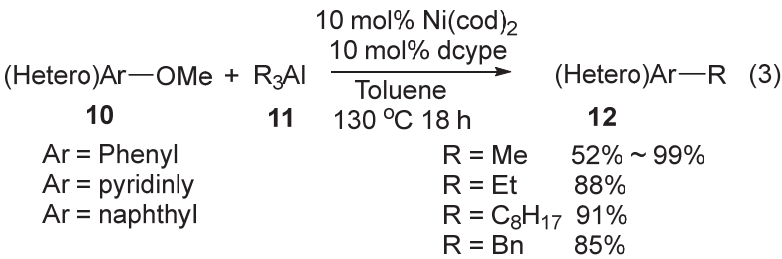

在另一项研究中, Nakajima 及其同事 ${ }^{[23]}$ 报道了镍催 化氯硅烷与烷基铝试剂的交叉偶联反应(Scheme 1). 采 用 $5 \mathrm{~mol} \% \mathrm{Ni}(\operatorname{cod})_{2} / 10 \mathrm{~mol}^{2} \% \mathrm{PCy}_{3}$ 络合物为催化剂, 1,4二氧六环为溶剂, 于 $60{ }^{\circ} \mathrm{C}$ 进行交叉偶联反应. 二氯硅 烷(13)与三烷基铝试剂(14)在该反应条件下反应 $20 \mathrm{~h}$, 以高选择性 $(15: \mathbf{1 6}=20: 1)$ 得到相应的偶联产物 $\mathbf{1 5}$. 化合物 15 在催化量 4-二甲氨基吡啶(DMAP)存在下与环 己醇和三乙胺反应，以 $11 \% \sim 99 \%$ 的收率得到相应的产 物 17. 三氯硅烷同样适用于该催化体系, 其相应产物 $\mathbf{1 7}$ 的收率为 $33 \% \sim 91 \%$.

\section{2 金属铜催化体系}

1978 年, Sato 和他的同事 ${ }^{[24]}$ 开发了烯丙基卤化物 (20)与烷基铝锂(19)的交叉偶联方法. 该方法在室温于 


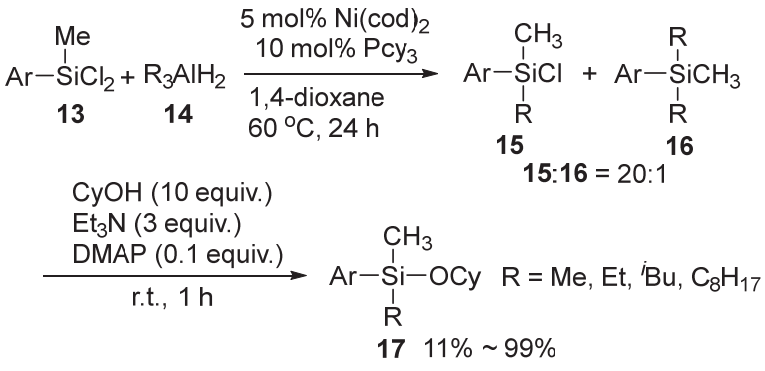

图式 1 镍催化氯硅烷与烷基铝试剂的交叉偶联反应

Scheme 1 Nickel catalyzed cross coupling reaction of chlorosilane with alkyl aluminides

四氢呋喃(THF)中, 用 $14 \mathrm{~mol} \% \mathrm{CuCl}$ 催化烯烃与 $\mathrm{LiAlH}_{4}$ 进行氢化铝化反应(Scheme 2). 各种有机铝试剂与 3-卤 代-1-丙烯进行交叉偶联反应, 在 $12 \mathrm{~h}$ 内以中等至良好 的收率 $(63 \% \sim 80 \%)$ 得到相应的偶联产物 21 . 当 $\mathrm{LiAl}-$ $\left(\mathrm{C}_{6} \mathrm{H}_{13}\right)_{4}$ 用于探索该反应对其它烯丙基卤化物的适用性 时, 各种烯丙基氯化物反应良好, 且能以中等的收率得 到相应的目标产物. 尤其值得注意的是, 具有多种功能 基团的烯丙基卤化合物均适用于该催化体系.

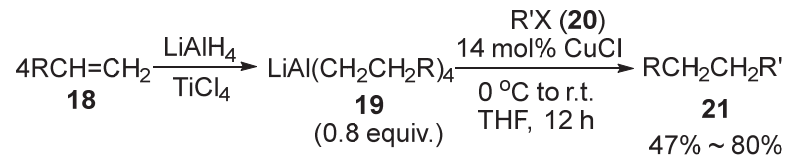

$$
\begin{aligned}
& \mathrm{R}=\mathrm{H}, \mathrm{CH}_{3}, n-\mathrm{C}_{4} \mathrm{H}_{9}, \mathrm{CH}_{3} \mathrm{CH}=\mathrm{CHCH}_{2}, \mathrm{CH}_{2}=\mathrm{C}\left(\mathrm{CH}_{3}\right) \mathrm{CH}_{2} \mathrm{CH}_{2} \text {, } \\
& \text { 2-cyclohexenyl }
\end{aligned}
$$

图式 2 铜催化烯丙基卤化物与烷基铝锂的交叉偶联 Scheme 2 Copper catalyzed cross-coupling of allyl halides with alkyl aluminum lithium

Giri 及其同事 ${ }^{[25]}$ 于 2016 年报道了铜催化的有机铝 试剂与有机卤化物的交叉偶联. 使用 $2 \sim 10 \mathrm{~mol} \% \mathrm{CuI} /$ 2 10 mol\% PN-1 (29, 图 2)作为催化剂, 在 DMF 中反 应 6 24 h, 以良好到优异的收率得到相应的偶联产物 (Eq. 6). 一级和二级烷基铝试剂均可用作该反应体系中 的金属有机偶联试剂. 烷基-芳基、芳基-芳基和芳基乙烯基偶联反应需要添加 PN-1 配体才能获得最佳的产 物收率. 然而, 有机铝试剂与杂芳基卤化物的偶联反应 不需要 PN-1 配体, 即使是一级和二级烷基铝试剂与杂 芳基卤化物的交叉偶联反应, 也不需要 PN-1 配体. 此 外, 这些反应不会因 $\beta-\mathrm{H}$ 的消除而变得复杂, 因此即使 在 “无配体” 条件下, 有机铝试剂与杂芳基卤化物进行 交叉偶联反应, 也不会在仲烷基铝试剂中观察到重排产 物. 根据实验结果, 作者提出了可能的反应机理(图 3).

\section{3 金属铁催化体系}

由于药物化学和农业化学中的 “烷基” 效应使得有<smiles>CN(C)c1ccccc1Pc1ccccc1</smiles>

图 2 PN-1 配体

Figure 2 PN-1 ligand

$$
\begin{aligned}
& \text { Aryl- } \mathrm{I}, \mathrm{Br} \quad 2 \sim 10 \mathrm{~mol} \% \mathrm{Cul} \quad \text { Aryl }-\mathrm{R} \\
& 23 \quad 2 \sim 10 \mathrm{~mol} \% \mathrm{Cul} \quad 26 \\
& 2 \sim 10 \mathrm{~mol} \% \text { PN-1 } 38 \% \sim 90 \% \\
& \mathrm{R}_{3} \mathrm{Al}+\operatorname{Aryl}(\mathrm{Het})-\mathrm{I}, \mathrm{Br}, \mathrm{Cl} \underset{\mathrm{LiCl}(3 \text { equiv. })}{\stackrel{\text { or no ligand }}{\longrightarrow}} \operatorname{Aryl}(\text { Het })-\mathrm{R} \\
& 22 \quad 24 \quad \mathrm{LiCl}(3 \text { equiv.) } 27 \\
& \text { (1 equiv.) } \\
& \mathrm{Ar} \sim \mathrm{Br} \\
& 25 \\
& \mathrm{Ar}_{28}^{\sim} \mathrm{R}
\end{aligned}
$$

图式 3 铜催化的有机铝试剂与有机卤化物的交叉偶联 Scheme 3 Copper catalyzed cross coupling of organoaluminides with organohalides

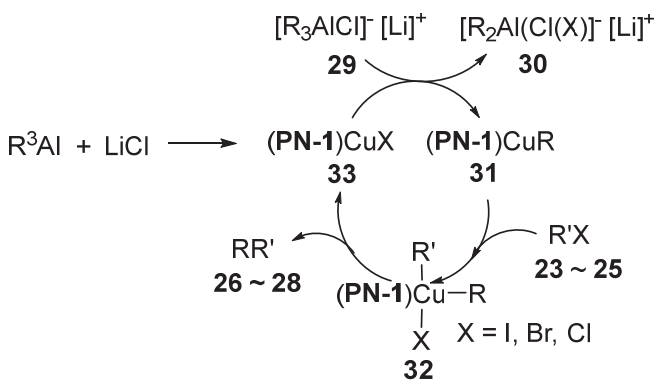

图 3 可能的催化循环

Figure 3 Proposed catalytic cycle.

机分子的烷基化反应受到广泛关注. Kotora 及其同 事 ${ }^{[26-27]}$ 发现了一种有趣的烷基化环化反应. 他们计划用 烷基铝试剂 $\left(\mathrm{R}_{3} \mathrm{Al}\right)$ 还原铁催化剂, 然后进行 2-氯庚烷1,6-二烯(34)的 Heck 型环化反应，得到烷基化产物 36, 而不是预期的偶联产物 37 (Eq. 4). 当使用 $\mathrm{AlMe}_{3}$ 时也产 生了同样的结果，作者提出了该反应可能的催化循环 (图 4).

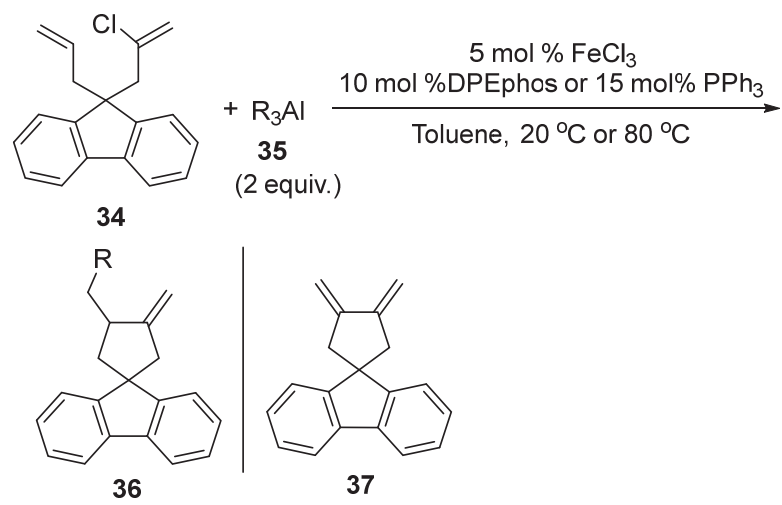

$20^{\circ} \mathrm{C} R=\mathrm{Me} 36 \%, \mathrm{R}=\mathrm{Et} 80 \%$ $80^{\circ} \mathrm{C} R=\mathrm{Me} 73 \%, \mathrm{R}=\mathrm{Et} 83 \%$

近年来, 通过直接 $\mathrm{C}-\mathrm{H}$ 活化将烷基引入分子中已 


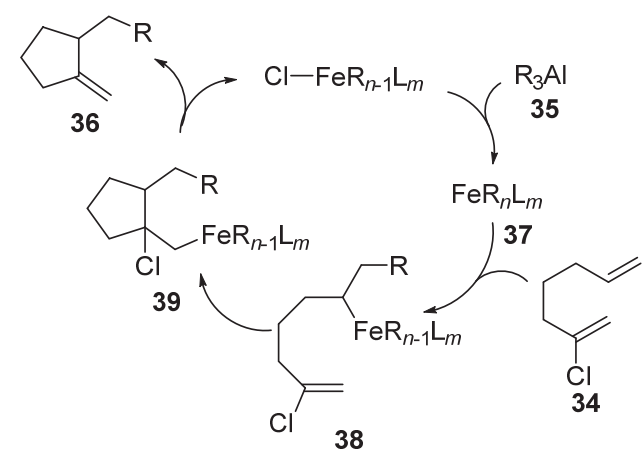

图 4 可能的催化循环

Figure 4 Proposed catalytic cycle

成为一个热点研究课题, 尽管这种方法非常困难 ${ }^{[28-32]}$. Nakamura 和他的同事 ${ }^{[33]}$ 报道了一种特别简单的用于三 甲基铝与含 8-氨基喹啉基的芳酰胺类化合物的邻位烷 基化反应，该反应用铁/二膦配体为催化剂，廉价的 2,3二氯丁烷为氧化剂(Eq. 5). 该催化体系的优点包括催化 体系简单，催化转化率高. 此外，该甲基化反应范围广、 选择性好, 对乙醚、三氟甲基、叔胺、酯、氟、氯等官 能团具有良好的耐受性, 对于具有与导向基处于邻位的 两个相同的 $\mathrm{C}-\mathrm{H}$ 键的底物, 其双甲基化是有利的. 重 要的是, 该反应可以成功地扩展到与三乙基铝的乙基化 反应，并且可以在该反应过程中使用空气稳定的双(三 甲铝 $) \cdot \mathrm{DABCO}(\mathrm{DABCO}=1,4-$ 二氮杂双环-[2.2.2]辛烷).

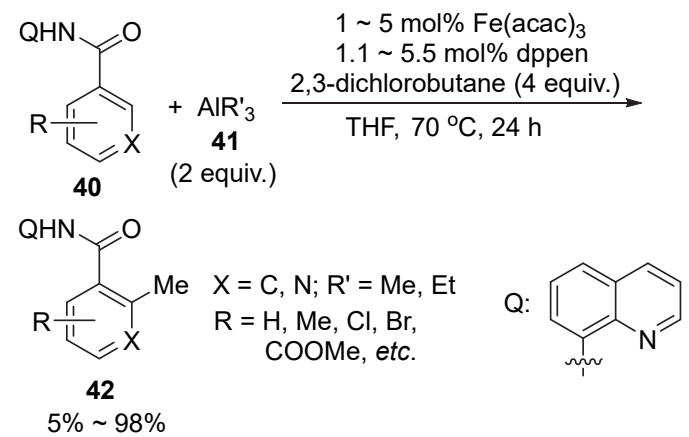

后来，底物被扩展到芳基酮和芳香羧酸、酯和酰胺. 他们发现, 在 $70{ }^{\circ} \mathrm{C}$ 下以 $5 \sim 10 \mathrm{~mol} \% \mathrm{Fe}(\mathrm{acac})_{3} / 5 \sim 10$ $\mathrm{mol} \%$ 三齿膦配体(37)(图 5)为催化剂, $\mathrm{THF} /$ 甲苯为溶剂, 2,3-二氯丁烷为氧化剂(图 6), 各种芳基酮和芳香羧酸、 酯和酰胺都可以与烷基铝试剂进行甲基化反应. 在标准 加热条件下, 所有交叉偶联反应均可在 $4 \mathrm{~h}$ 内完成, 甲 基化产物的收率高达 $98 \%$ (Eq. 6) ${ }^{[34]}$. 同时, 在某些情况 下, 还观察到竞争性二甲基化反应，通过增加催化剂负 载量和三甲基铝的用量, 可以获得较高收率的二甲基化 产物. 然而, 与三乙基铝的定向乙基化反应导致竞争性 的还原、加成和二聚反应. 各种官能团, 如硼酸酯、卤 化物、硫化物、杂环和烯醇化酮在这一过程中可以很好
地兼容.<smiles>CN(C)c1ccc(P(c2ccccc2)c2ccccc2-c2ccccc2)cc1</smiles>

图 5 三齿膦配体

Figure 5 Tridentate phosphine ligand

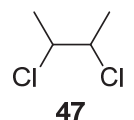

图 6 2,3-二氯丁烷

Figure 6 2,3-DCB

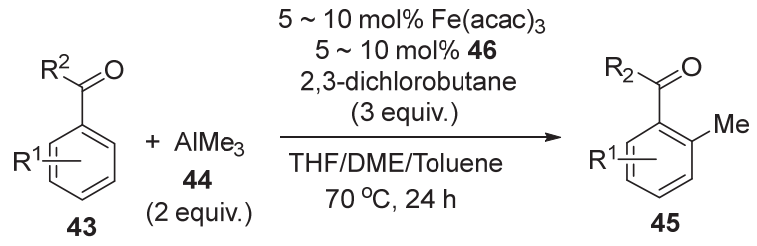

$\mathrm{R}^{1}=\mathrm{H}, \mathrm{MeO}, \mathrm{MeS}, \mathrm{F}, \mathrm{Cl}, \mathrm{Br}, \mathrm{CONH}$, etc. $\mathrm{R}^{2}=\mathrm{OH}, \mathrm{OMe}$, alkyl, etc.

\section{4 金属钴催化体系}

最近，使用廉价且丰富的过渡金属催化剂直接活化 $\mathrm{C}-\mathrm{H}$ 来构建碳一碳键已成为一个热门课题[35-37]. 研究表 明, 钴是完成此类转化的强有力催化剂[ [38-41], 用于 $\mathrm{C}-$ $\mathrm{H}$ 键与烷基有机金属试剂 ${ }^{[42-43]}$ 或烷基亲电试剂[44-46]的烷 基化反应.

2016 年, 徐坤和他的同事 ${ }^{[47-48]}$ 开发了一种简单的 三苯基膦搭配钴的催化剂, 以 2,3-二氯丁烷为氧化剂, 用于烷基铝试剂与含 8-氨基喹啉导向基的(杂)芳烃、烯 基甚至脂肪族碳酰胺的邻位 $\mathrm{C}-\mathrm{H}$ 键直接烷基化反应. 使用 $5 \mathrm{~mol} \% \mathrm{Co}(\mathrm{acac})_{2} / 5.5 \mathrm{~mol}^{2} \mathrm{PPh}_{3}$ 为催化剂, 1.2 equiv. 的 2,3-二氯丁烷作为氧化剂, THF 为溶剂, $80{ }^{\circ} \mathrm{C}$ 进 行反应(Eq. 7). 反应对芳烃上的供电子和吸电子取代基 的电子效应不敏感，对于甲氧基、胺基、氟、氯、三氟 甲基、二甲胺基、酯基等官能团具有良好的耐受性. 除 芳烃碳酰胺外, 杂环芳烃和脂肪族碳酰胺也是合适的反 应底物. 与之前建立的 $\mathrm{C}-\mathrm{H}$ 键的直接甲基化方法相比, 该体系显示出较好的单甲基化选择性. 此外，通过使用 1,10-菲咯啉作为钴的配体，可以以优良的单烷基化选 择性方式和良好的产率 $(81 \% \sim 86 \%)$ 实现 $\mathrm{C}-\mathrm{H}$ 键的乙 基化. 然而, 对于具有空间位阻的三烷基铝试剂, 如三 丙基铝和三异丁基铝，反应未能得到理想的烷基化产 物, 可能是空间位阻的原因. 与其它共催化的 $\mathrm{C}-\mathrm{H}$ 活 
化相比较 ${ }^{[37-41,49]}$, 并且结合实验结果, 作者推论这种单 烷基选择性可能通过图 7 所示的钴络合物 51 实现.

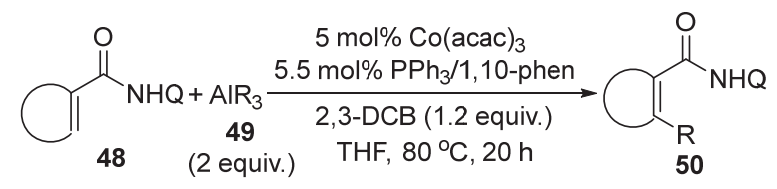

(hetero)aromatic, $71 \% \sim 93 \%$

alkenyl carboxamides

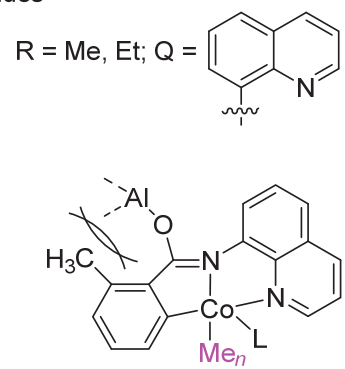

51

图 7 环状钴络合物中间体

Figure 7 A cobaltocycle intermediate

\section{5 金属铝催化体系}

烷基金属试剂与酰卤化合物的交叉偶联反应为酮 的合成提供了一种有效的方法. 1997 年, Nakagawa 和他 的同事 ${ }^{[50-51]}$ 报道了在 $\mathrm{AlCl}_{3}$ 存在下, 通过酰氯化物 $\mathbf{5 2}$ 与 三烷基铝(53)的交叉偶联反应, 有效地合成了 $\beta, \beta$-不饱 和酮(Eq. 8). 结果表明, 在室温反应 $2 \mathrm{~h}$, 可以以 $88 \%$ $100 \%$ 的收率得到酮的衍生物 54 . 此外, $\mathrm{RCOCl}$ 与 $\mathrm{R}^{\prime} \mathrm{AlCl}_{2}$ 或 $\mathrm{R}_{2}^{\prime} \mathrm{AlCl}$ 的交叉偶联反应也可以在不添加 $\mathrm{AlCl}_{3}$ 的情况下, 分别以 $100 \%$ 和 $77 \%$ 的收率得到相应的 RCOR'化合物.

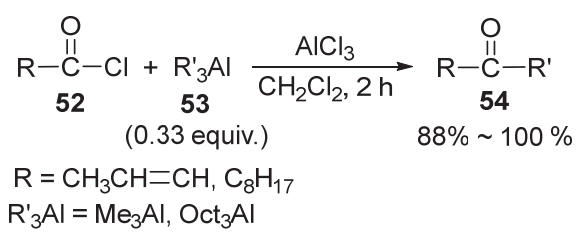

碳一氟键是化学反应中最惰性的化学键. 因此, 活 化 $\mathrm{C}-\mathrm{F}$ 键是一个挑战 ${ }^{[52]} .2009$ 年, Ozerov 和他的同 事 ${ }^{[53]}$ 开发了一种高效、长寿命的铝催化剂 $\mathrm{Et}_{2} \mathrm{Al}-$ $\left[\mathrm{HCB}_{11} \mathrm{H}_{5} \mathrm{Br}_{6}\right]$, 并评估了其在室温于正已烷溶液中对 $\mathrm{C}-\mathrm{F}$ 活化的催化活性(Scheme 4), 该催化剂对脂肪族 $\mathrm{C}-\mathrm{F}$ 键(脱氟烷基化)与烷基铝化合物的烷基化反应具 有良好的催化活性和选择性. 该催化剂对 $\mathrm{C}\left(\mathrm{sp}^{3}\right)-\mathrm{F}$ 键 有独特作用, 而 $\mathrm{C}\left(\mathrm{sp}^{2}\right)-\mathrm{F}$ 键则没有活化作用. 使用化 学计量的 $\mathrm{Me}_{3} \mathrm{Al}$ 作为反应试剂, 只将 $\mathrm{C}-\mathrm{F}$ 键转化为 $\mathrm{C}-\mathrm{Me}$ 键. 然而, 使用化学计量的 $\mathrm{Et}_{3} \mathrm{Al} 、{ }^{i} \mathrm{Bu}_{3} \mathrm{Al}$ 或 ${ }^{i} \mathrm{Bu}_{2} \mathrm{AlH}$ 作为反应试剂, 则会导致 $\mathrm{C}-\mathrm{F}$ 的取代与 $\mathrm{C}$ 一烷 基或 $\mathrm{C}-\mathrm{H}$ 的取代的竞争性反应. 尽管三烷基铝化合物
可以在不使用催化剂的情况下对反应性更强的底物的 $\mathrm{C}-\mathrm{F}$ 键进行活化，但催化剂可使反应速度更快，同时 提高反应的选择性. 重要的是，由于三烷基铝化合物可 作为一种清除剂, 因此该工艺过程可耐受少量水. 该反 应体系的优点是催化剂的寿命时间长, 周转次数高(可 达 $10^{4}$ 次).

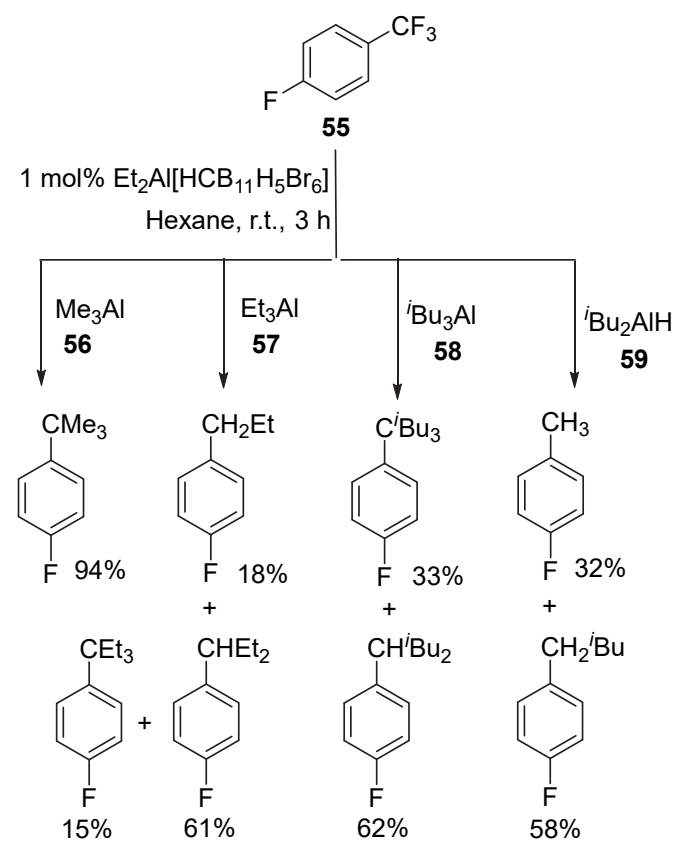

图式 $4 \mathrm{C}-\mathrm{F}$ 键参与烷基铝的偶联反应

Scheme $4 \mathrm{C}-\mathrm{F}$ bond participates in the cross-coupling reaction of alkyl aluminum

\section{2 无催化剂体系}

近年来，无催化剂条件下通过交叉偶联反应来构建 碳一碳键和碳一杂原子键方面取得了很大进展, 显示出了 巨大的发展前景 ${ }^{[54]}$. Yamamote 及其同事 ${ }^{[55]} 1976$ 年报 道了三烷基铝试剂与醇衍生物(60)在无催化剂条件下的 交叉偶联反应(Eq. 9). 在室温于正己烷溶液中，该反应 能以高达 $86 \%$ 的收率和高达 $97: 3$ 的化学选择性得到相 应的偶联产物 62. 虽然三烷基铝试剂与乙酸、甲酸酯、 碳酸酯的交叉偶联反应均能顺利进行，但是除三甲基铝 试剂能与四氢吡喃醚进行烷基化反应外, 其它的铝试剂 均不能有效地进行.

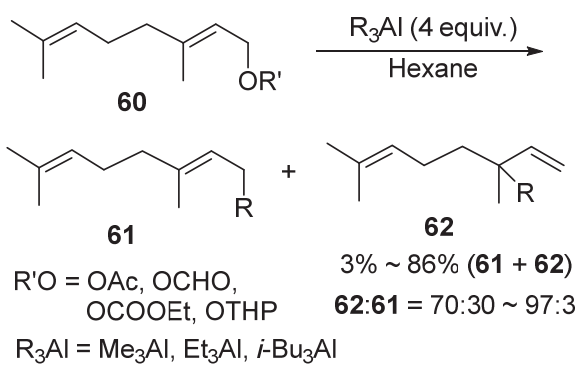


Maruoka 及其同事 [56]评估了无催化剂条件下有机 铝试剂和叔烷基氟化物的碳一碳键形成反应(Eq. 10). 在 没有外部亲核剂的情况下, 使用化学计量的三烷基铝试 剂可在温和的条件下, 通过从三烷基铝中的烷基转移来 实现简单的叔烷基化, 并以中等收率得到相应的偶联产 物 65. 此外, 烷基氟化物 63 与二甲基苯乙炔铝(1.5 equiv.)在甲苯溶液中 $-78{ }^{\circ} \mathrm{C}$ 反应 $30 \mathrm{~min}$, 能以 $70 \%$ 的 收率得到相应的偶联产物 65. 然而, 使用烷基氯化物未 能得到相应的偶联产物. 结果表明, 氟与铝之间的强相 互作用，是将惰性氟取代基转化为良好的离去基的必要 条件.

\begin{tabular}{|c|c|c|c|}
\hline$K_{63}$ & $\begin{array}{c}+\mathrm{RAIMe}_{2} \\
\mathbf{6 4} \\
\text { (1.5 equiv.) }\end{array}$ & $\frac{\text { solvent }}{-78^{\circ} \mathrm{C}, 30 \mathrm{~min}}$ & $K_{\mathrm{R}}{ }^{(1}$ \\
\hline $\mathrm{R}$ & $\mathrm{X}$ & Solvent & Yield/\% \\
\hline $\mathrm{Me}$ & $\mathrm{F}$ & $\mathrm{CH}_{2} \mathrm{Cl}_{2}$ & 70 \\
\hline Et & $\mathrm{F}$ & $\mathrm{CH}_{2} \mathrm{Cl}_{2}$ & 49 \\
\hline Hexanyl & $\mathrm{F}$ & $\mathrm{CH}_{2} \mathrm{Cl}_{2}$ & 48 \\
\hline $\mathrm{Ph}=\xi-$ & $\mathrm{F}$ & Toluene & 70 \\
\hline $\mathrm{Ph}=\xi-$ & $\mathrm{Cl}$ & Toluene & NR \\
\hline
\end{tabular}

后来, Terao 和 Kambe 等 ${ }^{[57]}$ 报道了使用有机铝试剂 作为亲核试剂的烷基化反应，也可以使用伯烷基氟化物 (Eq. 11). 在室温于正己烷溶液中, 烷基氟化物与铝试剂 无需使用催化剂, 便能以 $78 \%$ \% $99 \%$ 的收率得到相应偶 联产物 68. 在该反应体系中, 有机铝试剂很容易将烷基 氟化物的 $\mathrm{C}\left(\mathrm{sp}^{3}\right)-\mathrm{F}$ 键转化为 $\mathrm{C}\left(\mathrm{sp}^{3}\right)-\mathrm{X}(\mathrm{X}=\mathrm{Cl}, \mathrm{C}, \mathrm{H}$, $\mathrm{O}, \mathrm{S}, \mathrm{Se}, \mathrm{Te}, \mathrm{N})$ 键.

$$
\begin{aligned}
& n-\mathrm{C}_{8} \mathrm{H}_{17}-\mathrm{F}+\mathrm{RAIR}_{2} \underset{6}{\stackrel{\text { Hexane }}{\longrightarrow}} n-\mathrm{C}_{8} \mathrm{H}_{17}-\mathrm{R} \\
& \begin{array}{lll}
66 & 67 & 68
\end{array} \\
& \text { (1.2 } 1.5 \text { equiv. })
\end{aligned}
$$

\begin{tabular}{|c|c|c|c|}
\hline $\mathrm{R}_{2} \mathrm{Al}-\mathrm{R}^{\prime}$ & Time/h & Product & Yield/\% \\
\hline $\mathrm{Et}_{2} \mathrm{Al}-\mathrm{Et}$ & $1.5 r$ & $n-\mathrm{C}_{8} \mathrm{H}_{17}-\mathrm{Et}$ & 93 \\
\hline${ }^{i} \mathrm{Bu}{ }_{2} \mathrm{Al}-\mathrm{CH}=\mathrm{CH}^{n} \mathrm{Hex}$ & 6 & $n-\mathrm{C}_{8} \mathrm{H}_{17}-\mathrm{CH}=\mathrm{CH}^{n} \mathrm{Hex}$ & 90 \\
\hline $\mathrm{Et}_{2} \mathrm{Al}-\mathrm{C} \equiv \mathrm{C}-{ }^{n} \mathrm{Hex}$ & 16 & $n-\mathrm{C}_{8} \mathrm{H}_{17}-\mathrm{C} \equiv \mathrm{C}-{ }^{n} \mathrm{Hex}$ & 94 \\
\hline${ }^{i} \mathrm{Bu} 2 \mathrm{Al}-\mathrm{H}$ & 1.5 & $n-\mathrm{C}_{8} \mathrm{H}_{17}-\mathrm{H}$ & 97 \\
\hline${ }^{i} \mathrm{Bu}_{2} \mathrm{Al}-\mathrm{OPh}$ & 100 & $n-\mathrm{C}_{8} \mathrm{H}_{17}-\mathrm{OPh}$ & 78 \\
\hline${ }^{i} \mathrm{Bu} 2 \mathrm{Al}-\mathrm{SPh}$ & 1 & $n-\mathrm{C}_{8} \mathrm{H}_{17-\mathrm{SPh}}$ & 99 \\
\hline${ }^{i} \mathrm{Bu}_{2} \mathrm{Al}-\mathrm{SePh}$ & 1 & $n-\mathrm{C}_{8} \mathrm{H}_{17}-\mathrm{SePh}$ & 99 \\
\hline${ }^{i} \mathrm{Bu} 2 \mathrm{Al}-\mathrm{TePh}$ & 1 & $n-\mathrm{C}_{8} \mathrm{H}_{17}-\mathrm{TePh}$ & 92 \\
\hline${ }^{i} \mathrm{Bu}_{2} \mathrm{Al}-\mathrm{NEt}_{2}$ & 108 & $n-\mathrm{C}_{8} \mathrm{H}_{17}-\mathrm{NEt}_{2}$ & 85 \\
\hline
\end{tabular}

最近, Terao 和 Kambe 小组 ${ }^{[58]}$ 开发了在无催化剂条 件下使用有机铝试剂, 将一系列三氟甲基苯的 $\mathrm{C}-\mathrm{F}$ 键 转换为碳-碳键的有效方法(Eq. 12). 在混合溶剂己烷$\mathrm{CH}_{2} \mathrm{Cl}_{2}$ 中, $50{ }^{\circ} \mathrm{C}$ 下反应 $12 \mathrm{~h}$, 以 $81 \% \sim 89 \%$ 的收率得到
相应的偶联产物 69. 然而, 烷基铝试剂与 2-三氟甲基联 苯(72)反应，形成如 Scheme 5 所示的三种产物的混合 物. 这一结果可以解释为常见的苄基碳正离子中间体 73 经过直接甲基化、分子内 Friedel-Crafts 型反应或 $\beta-\mathrm{H}$ 消除反应转化为产物.
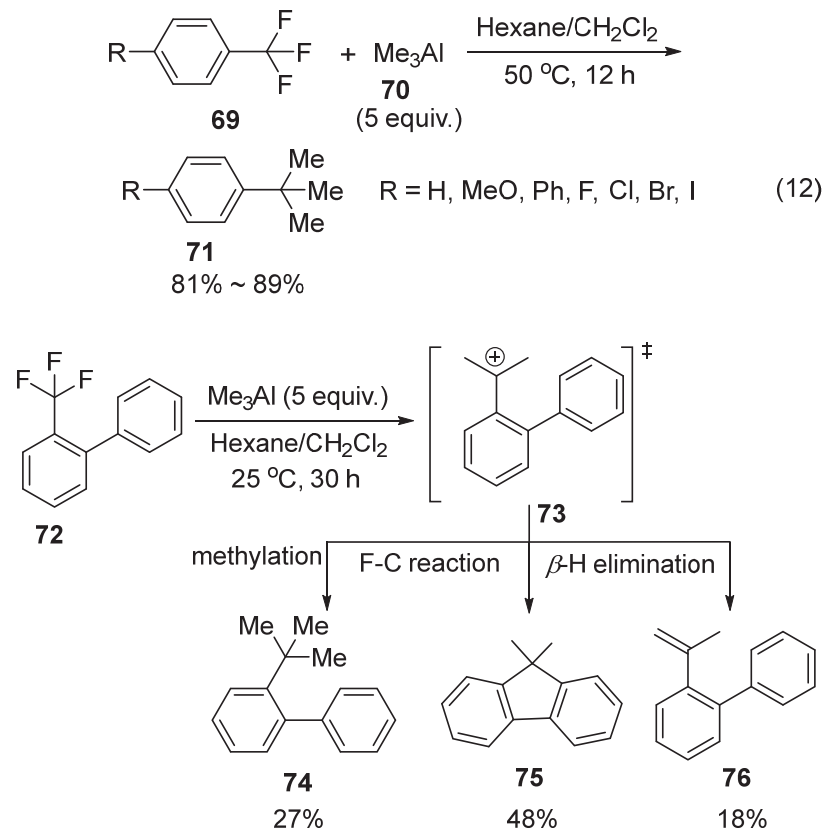

图式 5 无催化剂条件下有机铝试剂与三氟甲基苯的偶联反 应

Scheme 5 Cross-coupling reaction of organic aluminum reagent with trifluoromethylbenzene without catalyst

2007 年, Shishido 及其同事 ${ }^{[59]}$ 研究了无催化剂条件 下叔甲烷磺酸酯或卤化物与三甲基铝试剂的交叉偶联 反应(Eq. 13). 在 $\mathrm{CH}_{2} \mathrm{Cl}_{2}$ 或甲苯中, 1,1-二烷基-2,2,2-三 氟烷基衍生物(77)与三甲基铝试剂于室温反应 $1.5 \mathrm{~h}$, 以 $57 \% \sim 96 \%$ 的收率得到相应的偶联产物 78. 有趣的是, 使用二甲基氯化铝作为潜在的甲基源，能以 95\%的收率
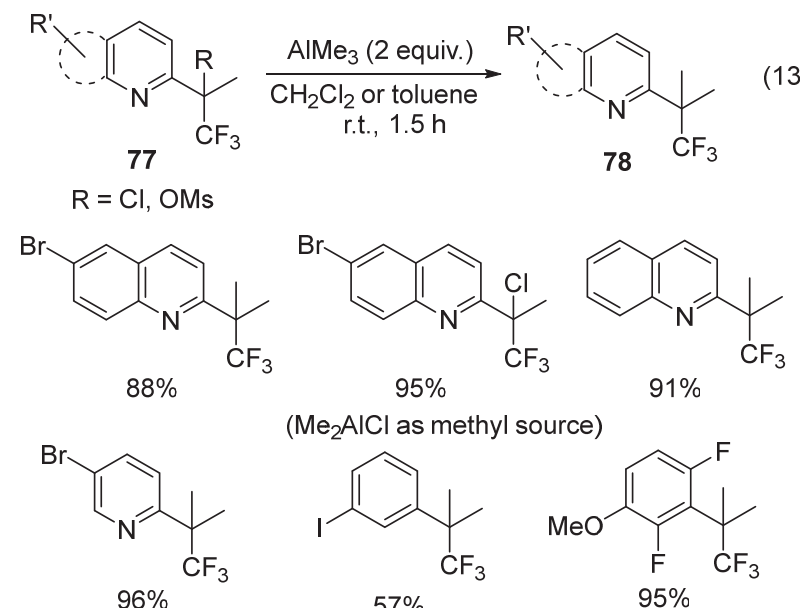

$57 \%$

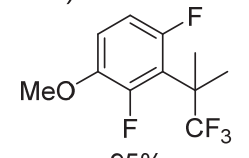

$\mathrm{R}=\mathrm{Cl}$, cyclohexane as solvent 
得到了氯代衍生物. 这些合成方法可用于 1,1 -二甲基2,2,2-三氟乙基化合物和类似物的合成，包括含芳香环 和杂环的化合物.

2010 年, Tanaka 及其同事[60]研究了 3'-脱氧胞嘧啶 的 4'-苯磺酰基衍生物与烷基铝试剂在无催化剂条件下 的交叉偶联反应(Eq. 14). 在优化的条件下, 各种 4'-取 代( Me, Et, ${ }^{i} \mathrm{Bu}, \mathrm{TMSC} \equiv \mathrm{C}$ )的类似物 79 与烷基铝试剂进 行偶联反应，能以 $62 \% \sim 81 \%$ 的收率和 $100 \%$ 的化学选 择性得到相应的偶联产物 80. 此外, 作者还证实了 4'苯磺酰基- $\beta-D$-底物的化学选择性优于 $\alpha-L$-异构体.

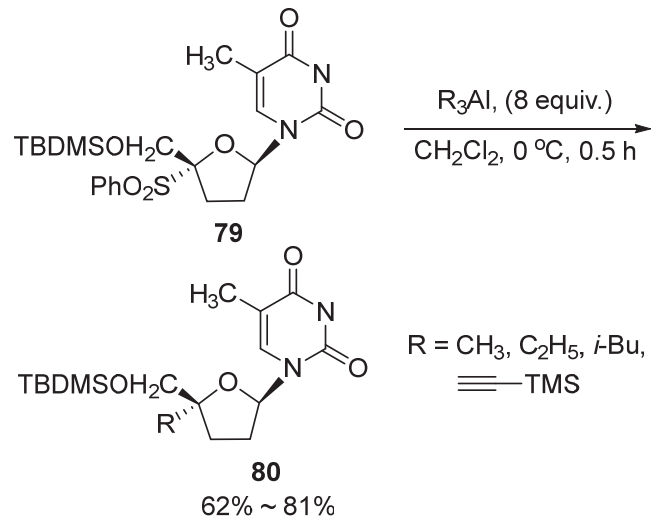

2011 年, Miyata 研究小组 ${ }^{[61]}$ 报道了一种在温和反应 条件下, 利用三烷基铝试剂作为亲核试剂, 通过 $N$-烷氧 基烯胺中间体实现酮的 $\alpha$-烷基化反应的策略. 在 $\mathrm{CH}_{2} \mathrm{Cl}_{2}$ 中, 室温反应 2 5 h 可获得良好的偶联产物 82 (Eq. 15). 作者对该反应体系提出了可能的反应机制 (Scheme 6).

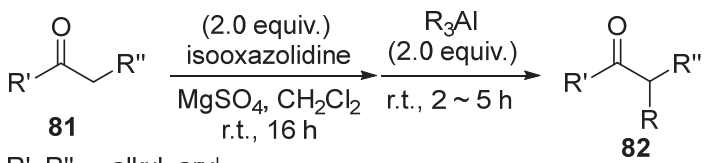

$\mathrm{R}^{\prime}, \mathrm{R}^{\prime \prime}=$ alkyl, ary

$\mathrm{R}=\mathrm{Me}, \mathrm{Et}, i-\mathrm{Bu} 44 \% \sim 78 \%$

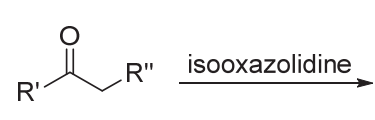

81<smiles></smiles>

85

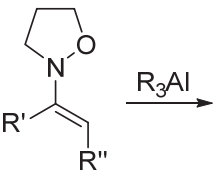

83

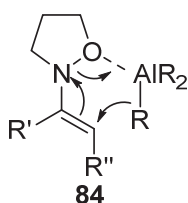

84<smiles>[R]C(=O)C([R])C</smiles>

图式 6 羰基化合物与三烷基铝试剂直接交叉偶联的可能机 理

Scheme 6 A possible mechanism of direct cross-coupling of carbonyl compounds with trialkyl aluminum reagents

异黄酮是一类广泛存在于豆科植物中的天然产物. 由于其具有多种生物活性 ${ }^{[62]}$, 使得异黄酮成为当前药

物化学合成的目标物之一[63-64]. 2015 年, Ohmori 和 Suzuki 小组 ${ }^{[65]}$ 报道了基于黄酮-3-甲基磺酸酯(86)中芳 基的 1,2-位迁移和有机铝试剂在原位进行烷基化反应来 立体选择性合成异黄酮化合物. 该反应在 $0{ }^{\circ} \mathrm{C}$ 下于 $\mathrm{CH}_{2} \mathrm{Cl}_{2}$ 中进行，无需金属催化，成功地以 $74 \%$ ～ $90 \%$ 的 收率合成了多种异黄酮衍生物 87 (Eq. 16). 此外, 该合 成方法已应用于 Isoflavan (-)-equol (88)的合成(图 9).

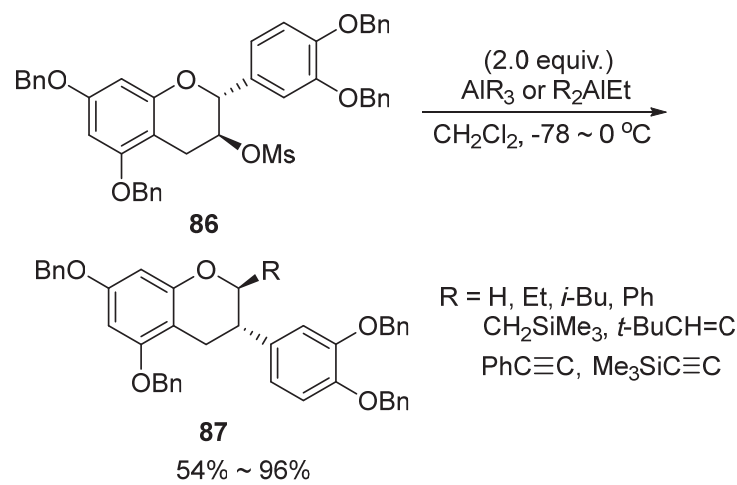<smiles>Oc1ccc([C@H]2COc3cc(O)ccc3C2)cc1</smiles>

图 9 异黄酮 $($ 一)等效物

Figure 9 Isoflavan (-)-equol

\section{3 结论与展望}

近十年来, 有机铝试剂在许多有机反应中得到了广 泛的应用，并取得了良好的效果．虽然以过渡金属为催 化剂或无金属催化条件下的有机铝试剂的交叉偶联反 应已经取得了长足的进展，但也存在一些不足. 比如金 属钯催化剂具有催化效率高、反应条件温和及底物范围 广的优点, 但是价格昂贵; 金属镍催化剂相较于金属钯 催化剂便宜, 但是一般反应温度较高, 时间长; 对于金 属铜、铁及钴催化剂, 它们适用的底物范围相较于金属 钯、镍要受限很多, 并且反应体系复杂一些. 为了解决 这些缺点, 还需要进一步研究它们的反应途径, 尽量减 少催化循环过程中过渡金属的浸出，探索铁、铜、镍等 其他环保型金属催化剂的应用. 因此,对有机铝试剂参 与的交叉偶联反应的研究，今后将重点集中在反应机理 的探讨，设计经济、高效的高性能催化剂和开发使用离 子液体、超临界流体等绿色溶剂作为替代有机溶剂等. 同时，利用微波、超声波、光催化或电化学[66]来促进该 类偶联反应的进行也是有待发展的一个重要研究领域.

\section{References}

[1] Negishi, E-i.; Zeng, X.; Tan, Z.; Qian, M.; Hu, Q.; Huang, Z. Palla- 
dium- or Nickel-Catalyzed Cross-Coupling with Organometals Containing Zinc, Aluminum, and Zirconium: The Negishi Coupling. In Metal-Catalyzed Cross-Coupling Reactions, Vol 2, WILEY-VCH Verlag GmbH \& Co. KGaA, Weinheim, 2004.

[2] Miyaura, N.; Suzuki, A. Chem. Rev. 1995, 95, 2457.

[3] Kang, S. K.; Yamaguchi, T.; Kim, T. H.; Ho, P. S. J. Org. Chem. 1996, 61, 9082 .

[4] Cherney, A. H.; Kadunce, N. T.; Reisman, S. E. Chem. Rev. 2015, $115,9587$.

[5] Herath, A.; Molteni, V.; Pan, S. F.; Loren, J. Org. Lett. 2018, 20, 7429

[6] Zhu, F.; Wang, Z. X. J. Org. Chem. 2014, 79, 4285

[7] Ranjan Jana, R.; Pathak, T. P.; Sigman, M. S. Chem. Rev. 2011, 111, 1417.

[8] (a) For the synthesis of organoaluminum compounds, see: Knochel, P.; Blîmke, T.; Groll, K.; Chen, Y.-H. Modern Organoaluminum Reagents: Preparation, Structure, Reactivity and Use. In Topics in Organometallic Chemistry, Vol. 41, Eds.: Woodward, S.; Dagorne, S., Springer, Berlin, 2013,pp. $173 \sim 186$.

(b) For cross-coupling reactions of organoaluminum compounds, see: Kolb, A.; Zezschwitz, P. Modern Organoaluminum Reagents: Preparation, Structure, Reactivity and Use. In Topics in Organometallic Chemistry, Vol. 41, Eds.: Woodward, S.; Dagorne, S., Springer, Berlin, 2013, pp. $267 \sim 276$.

[9] Maruoka, K.; Yamamoto, H. Tetrahedron 1988, 44, 5001.

[10] Varenikov, A.; Mark Gandelman, M. J. Am. Chem. Soc. 2019, 141, 10994.

[11] Li, Q. H.; Shao, X. B.; Ding, Y.; Wen, C.; Zhao, Z. G. Curr. Org. Chem. 2018, 22, 1523.

[12] Li, Q. H.; Wang, J. H.; Wen, C.; Xin, J.; Cao, K. P.; Wu, K.; Liang, M. Chin. Chem. Lett. 2019, 1830

[13] Li, Q. H.; Jiang, X.; Wu, K.; Luo, R. Q.; Zhang, Z. H.; Liang, M.; Huang, Z. Y. Mini-Rev. Org. Chem. 2020,

[14] Li, Q. H.; Shao, X. B.; Zhang, G.; Ding, Y.; Yang, X. J.; Chen, F. Chin. J. Org. Chem. 2018, 38, 802 (in Chinese). (李清寒, 杓学蓓, 张刚, 丁勇, 杨学军, 陈峰, 有机化学, 2018, 38, 802.)

[15] Magano, J.; Monfette, S. ACS Catal. 2015, 5, 3120.

[16] Tarui, A.; Shinohara, S.; Sato, K.; Omote, M.; Ando, A. Org. Lett. 2016, 18,1128

[17] (a) Matsubara, K.; Yamamoto, H.; Miyazaki, S.; Inatomi, T.; Nonaka, K.; Koga, Y.; Yamada, Y.; Veiros, L. F.; Kirchner, K. Organometallics, 2017, 36, 255.

(b) Gan, Y.; Zhang, N. H.; Huang, S. X.; Liu, Y. H. Chin. J. Chem. 2020, 38, 1686.

(c) Wang, H.; Wang, A. W.; Xia, Z. Z.; Zhou, W. Y.; Sun, Z. H.; Quan, J. F.; He, M. Y. Chin. J. Org. Chem. 2020, 40, 2099 (in Chinese). (王慧, 王安玮, 夏珍珍, 周维友, 孙中华, 钱俊峰, 何明阳, 有机 化学, 2020, 40, 2099.)

(d) He, S. J.; Pi, J. J.; Li, Y.; Lu, X.; Fu, Y. Acta Chim. Sinica 2018, 76, 956 (in Chinese).

(何世江, 皮静静, 李炎, 陆喜, 傅尧, 化学学报, 2018, 76, 956.)

[18] He, F.; Wang, Z. X. Tetrahedron 2017, 73, 4450

[19] Wang, D. Y, Kawahata M.; Yang, Z. K.; Miyamoto, K.; Komagawa, S.; Yamaguchi, K.; Wang, C.; Uchiyama, M. Nat. Commun. 2016, 7, 12937.

[20] Blum, J.; Gelman, D.; Baidossi, W.; Shakh, E.; Rosenfeld, A.; Aizenshtat, Z.; Wassermann, B. C.; Frick. M.; Heymer. B.; Schutte, S.; Wernik, S.; Schumann, H. J. Org. Chem. 1997, 62, 8681.

[21] Moria, Y.; Shigenob, C.; Luo, Y.; Chan, B.; Onodera, G.; Kimura, M. Synlett 2018, 29, 742 .

[22] Liu, C. Y.; Wititsuwannakul, T.; Hsieh, C. H.; Tsai, C. Y.; Wang, T. H.; Ambre, R.; Chen, W. C.; Surawatanawong, P.; Ong, T. G. J. Chin. Chem. Soc. 2020, 67, 376

[23] Naganawa, Y.; Guo, H. Q.; Sakamoto, K.; Nakajima, Y. ChemCatChem 2019, 11, 3756.

[24] Sato, F.; Kodama, H.; Sato, M. J. Organomet. Chem. 1978, 157, C30.

[25] Shrestha, B.; Thapa, S.; Gurung, S. K.; Pike, R. A. S.; Giri, R. J. Org.
Chem. 2016, 81, 787.

[26] Nečas, D.; Kotora, M.; Císařová, I. Eur. J. Org. Chem. 2004, 6, 1280.

[27] Nečas, D.; Drabina, P.; Sedlák, M.; Kotora, M. Tetrahedron Lett. 2007, 48, 4539.

[28] Chen, Q.; Ilies, L.; Yoshikai, N.; Nakamura, E. Org. Lett. 2011, 13, 3232.

[29] Chen, X.; Li, J.; Hao, X.; Goodhue, C. E.; Yu, J. Q. J. Am. Chem. Soc. 2006, 128,78 .

[30] Dai, H. X.; Stepan, A. F.; Plummer, M. S.; Zhang, Y.-H.; Yu, J. Q. J. Am. Chem. Soc. 2011, 133, 7222.

[31] Ilies, L.; Matsubara, T.; Ichikawa, S.; Asako, S.; Nakamura, E. J. Am. Chem. Soc. 2014, 136, 13126.

[32] Zhang, S. Y.; He, G.; Nack, W. A.; Zhao, Y.; Li, Q.; Chen, G. J. Am. Chem. Soc. 2013, 135, 2124.

[33] Shang, R.; Ilies, L.; Nakamura, E. J. Am. Chem. Soc. 2015, 137 , 7660 .

[34] Shang, R.; Ilies, L.; Nakamura, E. J. Am. Chem. Soc. 2016, 138, 10132 .

[35] Su, B.; Cao, Z. C.; Shi, Z. J. Acc. Chem. Res. 2015, 48, 886.

[36] Liu, W.; Groves, J. T. Acc. Chem. Res. 2015, 48, 1727.

[37] Tasker, S. Z.; Standley, E. A.; Jamison, T. F. Nature 2014, 509, 299.

[38] Moselage, M.; Li, J.; Ackermann, L. ACS Catal. 2016, 6, 498.

[39] Gao, K.; Yoshikai, N. Acc. Chem. Res. 2014, 47, 1208.

[40] Liang, Y.; Jiao, N. Angew. Chem., Int. Ed. 2016, 55, 4035.

[41] (a) Hummel, J. R.; Ellman, J. A. J. Am. Chem. Soc.,2015, 137, 490. (b) Gu, Z. Y.; Ji, S. J. Acta Chim. Sinica 2018, 76, 347 (in Chinese) (顾正洋, 纪顺俊, 化学学报, 2018, 76, 347.)

[42] Chen, Q.; Ilies, L.; Nakamura, E. J. Am. Chem. Soc. 2011, 133, 428

[43] Li, B.; Wu, Z. H.; Gu, Y. F.; Sun, C. L.; Wang, B. Q.; Shi, Z. J. Angew. Chem., Int. Ed. 2011, 50, 1109.

[44] Mei, R. H.; Ackermann, L. Adv. Synth. Catal. 2016, 358, 2443.

[45] Punji, B.; Song, W. F.; Shevchenko, G. A.; Ackermann, L. Chem.-Eur. J. 2013, 19, 10605

[46] Gao, K.; Yoshikai, N. J. Am. Chem. Soc. 2013, 135, 9279.

[47] Wang, H. Q.; Zhang, S.; Wang, Z. Q.; He, M. H.; Xu, K. Org. Lett. 2016, 18,5628 .

[48] Xu, K.; Tan, Z. M.; Zhang, H. N.; Zhang, S. Synthesis 2017, 3931.

[49] Li, Q.; Li, Y. R.; Hu, W. P.; Hu, R. J.; Li, G. G.; Lu, H. J. Chem.-Eur. J. 2016, 22, 12286.

[50] Arisawa, M.; Torisawa, Y.; Nakagawa, M. Synthesis 1995, 1371

[51] Arisawa, M.; Torisawa, Y.; Kawahara, M.; Yamanaka, M.; Nishida, A.; Nakagawa, M. J. Org. Chem., 1997, 62, 4327.

[52] (a) Amii, H.; Uneyama, K. Chem. Rev. 2009, 109, 2119.

(b) Ren, Z. W.; Ren, N.; Zhang, F. G.; Ma, J. A. Acta Chim. Sinica 2018, 76, 940 (in Chinese).

(任智雯, 任楠, 张发光, 马军安, 化学学报, 2018, 76, 940.)

[53] Gu, W. X.; Haneline, M. R.; Douvris, C.; Ozerov, O. V. J. Am. Chem. Soc. 2009, 131, 11203.

[54] (a) Dong, D. Q.; Li, G. H.; Chen, D. M.; Sun, Y. Y.; Han, J. J.; Wang, Z. L.; Xu, X. M.; Yu, X. Y. Chin. J. Org. Chem. 2020, 40, 1766 (in Chinese).

(董道青, 李光辉, 陈德茂, 孙媛媛, 韩晴晴, 王祖利, 徐金明, 于 贤勇, 有机化学, 2020, 40, 1766.)

(b) Li, Y. P.; Wang, M.; Jiang, X. F. Chin. J. Chem., 2020, 38, 1521

[55] Hashimoto, S.; Kitagawa, Y.; Iemura, S.; Yamamote, H.; Nozaki, H. Tetrahedron Lett. 1976, 30, 2615.

[56] Ooi, T.; Uraguchi, D.; Kagoshima, N.; Maruoka, K. Tetrahedron Lett. 1997, 38, 5679.

[57] Terao, J.; Begum, S. A.; Shinohara, Y.; Tomita, M.; Naitoh, Y.; Kambe, N. Chem. Commun. 2007, 855.

[58] Terao, J.; Nakamura, M.; Kambe, N. Chem. Commun. 2009, 6011.

[59] Tanaka, H.; Shishido, Y. Bioorg. Med. Chem. Lett. 2007, 17, 6079.

[60] Shimada, H.; Kikuchi, S.; Haraguchi, K.; Tanaka, H. Carbohydr. Res. 2010, 345, 2616.

[61] Miyoshi, T.; Miyakawa, T.; Ueda, M.; Miyata, O. Angew. Chem., Int. Ed. 2011, 50, 928 .

[62] Smith, D. A.; Banks, S. W. Phytochemistry 1986, 25, 979.

[63] Goel, A.; Kumar, A.; Raghuvanshi, A. Chem. Rev. 2013, 113, 1614. 
[64] Feng, Z. G.; Bai, W. J.; Pettus, T. R. R. Angew. Chem., Int. Ed. 2015, $54,1864$.

[65] Nakamura, K.; Ohmori, K.; Suzuki, K. Chem. Commun. 2015, 51, 7012.

[66] (a) Shuai Liu, S.; Li, J.; Wang, D. L.; Liu, F.; Liu, X.; Gao, Y. Y.; Jie, D.; Cheng, C. Chin. J. Chem. 2019, 37, 570.

(b) Dong, K.; Liu, Q.; Wu, Li. Z. Acta Chim. Sinica 2020, 78, 299 (in Chinese).
(董奎, 刘强, 吴俩珠, 化学学报, 2020, 78, 299.)

(c) Ma, X. D.; Zhang, G. Z. Chin. J. Chem. 2020, 38, 1299.

(d) Chen, Y. F.; Zhao, H.; Cheng, D. P.; Li, X. N.; Xu, X. L. Chin. J. Org. Chem. 2020, 40, 1297 (in Chinese).

(陈跃峰，赵赫，程冬萍，李小年，许孝良，有机化学，2020，40, 1297.)

(Fan, Y.) 